\title{
LA CONSTRUCGIÓN DE LA COMUNIDAD Y LA TOMA DE DECISIONES MORALES EN ENTORNOS DIGITALES. EL CASO DEL VIDEOJUEGO THIS WAR OF MINE
}

\author{
Emiliano ALDEGANI \\ (UNMdP, CONICET)
}

Gabriel DETCHANS

(UNMdP)

\section{RESUMEN}

El presente trabajo busca recuperar determinados lineamientos discursivos que pueden identificarse en torno a las formas de asociación de individuos y grupos en contextos hostiles, así como sobre la jerarquización axiológica que se observa en videojuegos del género survival, en particular, en el juego This War of Mine, sobre los elementos que componen el desarrollo de las prácticas sociales actuales. La presentación de la formación del sentido comunitario y las tensiones que se generan en contextos de hostilidad y precariedad como el escenario que propone este título, remiten a formas de agrupamiento entre distintos civiles en el marco de un conflicto bélico. El análisis del discurso ideológico que presenta el videojuego se realizará destacando la resignificación que propone de elementos asociados a la vida urbana a partir de la función que les es otorgada en el marco de la dinámica videolúdica. Es decir, en la medida que estos elementos pueden combinarse y agenciarse para desarrollar un recorrido dentro del entorno de juego que conduzca a las condiciones de victoria que el juego propone.

Palabras Clave: Comunidad-Alteridad- Videojuegos- Guerra

\section{ABSTRACT}

The present work seeks to recover from a critical perspective the discursive guidelines that can be identified around the forms of association of individuals and groups in hostile contexts, as it is presented in video games of the survival genre, in particular, in the game This War of Mine. The presentation of the formation of the community sense and the tensions that 
are generated in contexts of hostility and precariousness as the scenario proposed by this title, refer to the forms of grouping that occur between different civilians in the context of a warlike conflict. The analysis of the ideological discourse presented by the videogame will be carried out highlighting the resignification that it proposes of elements associated with urban life based on the function that is granted to them within the videoludic dynamics. That is to say, to the extent that these elements can be combined and organized to develop a journey within the game environment that leads to victory conditions.

Keywords: Comunnity- Otherness- Videogames- War

\section{INTRODUCCIÓN}

El presente trabajo busca recuperar desde una perspectiva crítica los lineamientos discursivos que pueden identificarse en torno a las formas de asociación de individuos y grupos en contextos hostiles, tal como es presentada en videojuegos del género survival, en particular, en el juego This War of Mine. La presentación de la formación del sentido comunitario y las tensiones que se generan en contextos de hostilidad y precariedad como el escenario que propone este título, remiten a las formas de agrupamiento que se producen entre distintos civiles en un el marco de un conflicto bélico. El análisis del discurso ideológico que presenta el videojuego se realizará destacando la resignificación que propone de elementos asociados a la vida urbana a partir de la función que les es otorgada al interior de la dinámica videolúdica. Es decir, en la medida que estos elementos pueden combinarse y agenciarse para desarrollar un recorrido dentro del entorno de juego que conduzca a las condiciones de victoria.

En este sentido, el artículo recuperará de forma explícita los trabajos de Manuel Sicart y su comprensión del entorno videolúdico como un escenario problemático que puede presentar a los jugadores dilemas éticos relevantes y que asigna una determinada valoración moral a los existentes que evoca, así como los trabajos de Óliver Pérez Latorre y lan Bogost sobre la posibilidad de recuperar la estructura semántica del entorno videolúdico como un discurso que comporta elementos ideológicos y persuasivos que establecen un diálogo con el imaginario social occidental desde el que se proyectan.

En consecuencia, el trabajo buscará explicitar el modo particular en el que se representan las relaciones sociales dentro del juego, atendiendo igualmente al modo en que son pensadas las formas de asociación en el contexto de un conflicto militar, pero también en el marco de relaciones hostiles de competencia y de precariedad que pueden asociarse a formas actuales de producción y de interacción que solo son exaltadas en el marco de un escenario distópico como el que presenta el juego.

A su vez, siguiendo la perspectiva de Antonio Planells y su apropiación de herramientas conceptuales de la narratología de Lubomír Doležel y Algirdas Greimas para la comprensión y el análisis de la construcción de sentido en el discurso videolúdico, el trabajo buscará explicitar el vínculo que se establece entre el mundo ludoficcional que propone This War of mine y los campos de referencia con los que su trasfondo o playwolrd (Frasca: 2009) entra en diálogo. De manera que la semántica intencional y los existentes que presenta el juego puedan ser pensados en su vínculo con un marco o semántica extensional vinculada a los conflictos militares de Sarajevo, Aleppo y la Segunda Guerra Mundial.

A fin de realizar el análisis propuesto, el trabajo se desarrollará en un primer apartado en el que se recuperarán algunas observaciones de la filosofía crítica, en particular de autores 
como Cornelius Castoriadis, en relación con la construcción de los espacios comunitarios y las problemáticas asociadas a la noción de comunidad. Seguido de un apartado en el que se abordará propiamente cómo estas problemáticas y tensiones son presentadas en This War of Mine y el modo en que puede recuperarse la valoración que propone el juego de los elementos que presenta, al observar el lugar que ocupan en relación a la mecánica de juego. Seguidamente, el trabajo expondrá algunas particularidades de This War of Mine en relación con su potencial como juego de representación histórica y el potencial del discurso audiovisual e interactivo para la reconstrucción de procesos históricos. Para ello se realizará un análisis comparativo de algunos elementos que se presentan en el juego y el campo de referencias al que remiten. Finalmente, se ofrecerán algunas observaciones sobre el discurso ideológico imperante en el entorno de juego, que pueden desprenderse de aspectos particulares de la mecánica que propone y que pueden entrar en fricción con el esquema narrativo general que el juego parece construir. Esta fricción entre la mecánica de juego y el esquema simbólico permitirá observar algunas líneas del imaginario social imperante en la actualidad, vinculado a la maximización de los procesos de producción y al afán de lucro, que ofrecen una perspectiva del discurso ideológico y la valoración moral que el juego propone de determinados elementos y su lugar en el marco general de la producción cultural.

\section{COMUNIDAD Y CONFLICTIVIDAD SOCIAL}

Atendiendo la perspectiva de las Ilamadas filosofías críticas de segunda mitad del siglo XX como las ideas propuestas por Cornelius Castoriadis, se advierte que, en la formación de un espacio comunitario, las formas de vincularse de la interacción social se orientan en torno de dos ejes que estructuran en gran medida el comportamiento grupal. A saber, la emergencia de las nociones de dentro y fuera del grupo, que define la pertenencia a lo común, y por otra parte, la naturalización de lo contingente dado en el momento inicial.

La formación de un grupo social comporta una clausura respecto de la exterioridad, pero esta exterioridad puede pensarse de diferentes maneras. Por un lado, lo exterior a la comunidad se identifica con los individuos que no pertenecen a ella, pero fundamentalmente, el exterior de la comunidad o de un grupo social, surge con el contacto con otros grupos sociales. No es el individuo como tal el que resulta una alteridad $u$ otredad a priori sino el conjunto de prácticas, símbolos y modos de hacer que conforman su identidad, y que se asocian con el imaginario de otro grupo de individuos y sus costumbres. Por otro lado, en un sentido más profundo, la comunidad implica una clausura respecto de su propio devenir, y de la alteridad que emerge dentro de sí por el mismo carácter extático del tiempo (Castoriadis 2005: 17). Las prácticas y significaciones instituidas en el interior de la sociedad tienden a clausurarse sobre sí mismas, a proyectarse la forma de todo tiempo pasado y de todo porvenir, y en este sentido comportan una naturalización de las condiciones iniciales en tanto que se interpreta a un conjunto arbitrario de disposiciones como necesario, natural o intrínsecamente bueno.

Esta clausura del grupo sobre su marco simbólico y la naturalización de sus prácticas otorga al conjunto un grado de unidad en su forma de hacer y representar. La identidad que se construye en torno al marco simbólico-normativo del cuerpo social constituye una forma de apropiación de la experiencia colectiva, y a la vez lo que autores como Castoriadis denominarán la creación de un mundo propio para el cuerpo social (Castoriadis 2010: 300312). Un mundo que surge en la apropiación que cada sociedad establece de un conjunto 
de condicionamientos naturales a partir de las formas institucionales que la organizan. El imaginario social que envuelve a un grupo o comunidad posee la propiedad de ser saturante, es decir, de otorgar un sentido a todo aquello que puede surgir en el seno de la vida social, y que reduce el carácter inquietante de lo emergente, otorgándole un sentido de antemano.

Sin embargo, si se producen cambios significativos en el entorno donde se desarrolla la vida social, el imaginario social que organiza sus prácticas y sus representaciones puede entrar en crisis obligando a los individuos a resignificar aquellos elementos que se han modificado y no pueden ser apropiados de un modo coherente desde el marco simbólico en el que los individuos se han formado.

El contexto particular que propone como escenario el videojuego que se analiza en este trabajo se corresponde, sin duda, con este tipo de alteraciones del curso de la vida que ponen en crisis todo el conjunto de nociones, valoraciones y formas de significar el entorno por parte de los individuos. En This War of Mine nos encontramos en un contexto de guerra donde la población civil debe encontrar el modo de sobrevivir a un entorno hostil, en el que deberán encontrar nuevas formas de asociarse y apropiarse de las existencias que propone el juego. La red de significaciones mediante la que los personajes configuran sus representaciones del entorno y organizan su forma de proyectarse en la acción, se ve alterada por un estado de total excepcionalidad, en el que, sin embargo, pueden observarse ecos del imaginario social en el que se han formado como individuos, pero que entran ahora en fricción con un nuevo marco de posibilidades. Los individuos se agrupan y organizan en torno a formas provisionales de hacer, pues el tiempo de excepción en el que se encuentran inmersos no les permite instituir o configurar relaciones que puedan devenir regularidades reales.

La capacidad de los hombres de crear se ve obstruida por un estado de extrema necesidad, que busca conservar aquellas condiciones iniciales hasta su restitución. En este sentido afirma Castoriadis: "aún en las situaciones de crisis, aún en medio de conflictos interiores y de las guerras intestinas más violentas, una sociedad continúa siendo esa misma sociedad; sino lo fuera no podría haber lucha alrededor de los mismos objetos, objetos comunes» (Castoriadis 2005: 68). La búsqueda de supervivencia de los personajes dentro de este marco estará supeditada a la capacidad de resignificar diversos elementos de su vida anterior, en relación con su potencial de combinación y de utilización en un nuevo marco, pero tendrá sin embargo como punto de inicio el imaginario precedente que aparece en el entorno de juego de diferentes maneras.

\section{CONSTRUCCIÓN DEL CONCEPTO DE COMUNIDAD EN THIS WAR OF MINE:}

El videojuego This War of Mine, lanzado en 2014 por la compañía polaca 11 bit Studios, coloca al jugador en la situación de administrar las acciones de un grupo de supervivientes civiles atrapados en un conflicto militar en una ciudad sitiada en el mundo contemporáneo. A diferencia de la mayoría de los juegos del tipo bélico, las acciones que propondrá la dinámica de juego no estarán vinculadas a acciones militares, de despliegue táctico o de utilización de armamentos y maquinarias militares, sino por el contrario, a la obtención y administración de los elementos necesarios para la supervivencia. Esto no quita que existan en el juego acciones ofensivas o incluso la utilización de armas, pero su presencia no está vinculada a un uso militar, sino a la aparición de elementos que forman parte del clima de hostilidad y peligro del entorno.

Quaderns, 13 (2018), pp. 23-36 
This War of Mine aparece en el contexto de los juegos con temática bélica como un juego de gestión de recursos donde la escasez (austeridad) fuerza al jugador a tomar decisiones difíciles de carácter ético, como podría ser decidir quién de dos supervivientes enfermos obtiene la única dosis de medicina disponible o si se está dispuesto a robar la última comida de un anciano refugiado en otro edificio para alimentar a un niño que forma parte del propio grupo. A la perspectiva lúdica obligatoria de cada juego se le suma, en este caso, cierta perspectiva persuasiva en la que se pretende generar empatía por parte del jugador hacia la difícil situación que atraviesa la población civil de una zona en guerra. En este sentido, el conjunto de reglas que estructura el juego opera, como afirma Miguel Sicart, como agentes semánticos que otorgan un valor y una relevancia a cada uno de los elementos, al introducirlos en el marco de un sistema de bonificaciones y penalizaciones, que en este caso se identifican con los elementos o sucesos que aseguran la supervivencia del grupo, o aquellos que la obstaculizan (Sicart 2009: 24), pero también con el significado que se otorga a las acciones en relación a los elementos para-textuales a los que están vinculadas en el juego.

Los elementos que conforman el clima general del juego se caracterizan por generar un ambiente opresivo, en el que la tensión emocional forma parte esencial de la jugabilidad y cada decisión comporta consecuencias difíciles de anticipar para el jugador.

Sin embargo, This War of Mine no emite un juicio de valor explícito sobre las elecciones tomadas por el jugador, dejándole decidir por sí mismo la manera más apropiada de realizar su experiencia de juego, aunque el jugador puede advertir rápidamente que la moral de los personajes muestra distinto grado de tolerancia a las acciones violentas. Esto diferencia a los personajes y el jugador puede hacerse una idea del impacto de los acontecimientos consultando el diario personal de cada personaje, del que se infiere fácilmente la aprobación o desaprobación de lo que ha sucedido, y esta aprobación cobra relevancia dentro de la gameplay, pues si los personajes se ven emocionalmente afectados por los acontecimientos pueden caer en un estado de depresión que requerirá la contención del grupo y, a su vez, puede ocasionar conflictos entre ellos, peleas que provoquen heridas en uno de los personajes e incluso si la situación se vuelve intolerable para ellos pueden llegar a suicidarse. A su vez, por encima de la gameplay específica del juego y la mecánica que propone, al finalizar una partida, si se ha alcanzado el final de la guerra el juego ofrece una breve descripción del destino del personaje en el que éste puede reponerse o no de aquello que ha atravesado en la guerra, según las decisiones que haya tomado el jugador. Como se anticipa en el trailer del juego, "Las cosas que haces en la guerra te acompañan por siempre». La posibilidad del personaje de reponerse de los acontecimientos que tienen lugar en el juego comporta un límite al cálculo estrictamente pragmático con el que puede desarrollarse la partida.

En efecto, el personaje/jugador como lo denomina Pérez Latorre aparece en This War of Mine como un emergente en la interacción entre la moral del jugador y el esquema moral que el juego propone. Existe en el playworld propuesto por el juego una dimensión pragmática en la que el jugador debe decidir si utilizar a un personaje para contener emocionalmente a otro, o para fabricar objetos que puedan vender para conseguir alimentos atendiendo a las necesidades y posibilidades del conjunto, pero también desde una perspectiva moral, pues la valoración de ayudar a uno de los integrantes del grupo tiene un efecto emocional. Estas dos dimensiones atraviesan todo el marco de decisiones que propone el juego. Si bien en un momento dado puede resultar mejor, desde una perspectiva práctica, utilizar a 
todos los personajes disponibles para producir, fabricar y comerciar, la misma mecánica del juego obliga a atender a otras necesidades en tanto que el estado anímico de los personajes puede conducir a un derrumbe moral del grupo que finalmente obstaculice la supervivencia del conjunto.

Ahora bien, incluso cuando el playworld que construye el juego sitúa a los jugadores en un contexto particular que es poco probable que haya sido vivenciado previamente por ellos, la moral o el imaginario general asociado a los personajes que éste debe orientar en el juego no difiere del imaginario social relativo a un individuo perteneciente al mundo contemporáneo. La construcción del sujeto-jugador en el This War of Mine busca posicionar al jugador en el lugar de un grupo de individuos, con los que puede compartir determinadas valoraciones morales respecto de lo correcto y lo incorrecto, pero en el contexto de un estado de completa excepción. El marco simbólico-normativo que orienta a los personajes en relación a sus acciones no está constituido en el contexto en el que deben desenvolverse, y las situaciones que deben afrontar no han sido contempladas en el marco simbólico en el que se han formado. En este sentido, las situaciones que deben atravesar los personajes resultan novedosas por igual al jugador y a los personajes. Existen en el juego múltiples referencias a los tiempos anteriores a la guerra y al momento en que la guerra termine y su mundo, tal como lo conocen los jugadores y los personajes, se reestablezca.

Por ejemplo, al pararse frente a un hotel en ruinas uno de los personajes dice «pensar que alguna vez brillaba y ahora parece una sombra de sí mismo», o al encontrarse frente a una casa afirma: «cuando todo esto termine compraré una casa como ésta». En el diario de uno de los personajes aparece la anotación "Ya lloraremos estas acciones cuando termine la guerra». Advierte la inmoralidad de algunas acciones, pero también su necesidad para sobrevivir hasta que se restablezca el orden social en que estas acciones sean sancionables.

La construcción del esquema moral que propone el juego está vinculada a un estado de excepción en el que es necesario resignificar y revalorizar los elementos y acciones que se han valorado y a los que se les ha otorgado un significado en el tiempo. En este sentido existe un vínculo estrecho entre el esquema moral del juego y una comprensión excepcional de la temporalidad que se experimenta durante la guerra, en la que se construye un juego de interacciones y constantes asociaciones entre una moral previa al estado de excepción y la emergencia de una nueva jerarquización axiológica del entorno que no puede instituirse o consolidarse como una nueva moral.

El tiempo en el que el juego trascurre se presenta como una realidad de carácter excepcional, donde los elementos que componen esa realidad cobran nuevos significados que deben ser explorados, establecidos y descubiertos por el jugador. ¿Qué importancia tendrá el agua potable? ¿Qué efecto puede tener en un marco de tal precariedad la falta de cigarrillos, vino, libros y otros objetos similares? ¿Qué limites cabe trasgredir para asegurar la supervivencia? La reapropiación y revalorización de estos elementos conocidos tanto por el jugador como por los personajes, se presenta como oportunidad para explorar el contexto de la guerra desde una perspectiva axiológica. ¿Cómo se integran nuestras valoraciones en un contexto de crisis, peligro y de difícil subsistencia? ¿Cuál es la relación que cabe establecer con otros grupos de sobrevivientes? La exploración que propone This War of Mine no está vinculada a que nos familiaricemos con elementos militares al modo de otros juegos con temáticas asociadas a la guerra tales como Battlefield 1942 (EA Digital Illusions, 2002) o Call of Duty (Infinity Ward, 2003) en los que exploramos el campo de batalla descubriendo armamentos y sus potenciales para la defensa y el ataque. Más 
allá de la presencia de algunos armamentos, el playworld que propone This War of Mine está compuesto por un conjunto de existentes que se vinculan de manera primordial con la vida civil ordinaria dentro de una ciudad. Existe, en las imágenes que aparecen entre escenas e incluso en el fondo de la pantalla de juego, la presencia de tanques de guerra u otros elementos militares, pero la mayor parte de los elementos están vinculados al mundo anterior a la guerra.

Incluso los soldados son resignificados en el entorno que los personajes exploran, pues sin importar la facción a la que pertenezcan representan un peligro para los civiles.

A la vez el juego enfatiza cierta debilidad moral por parte del ejército, y un grado de anomia, en tanto que puede ofrecer armas a los civiles a cambio de bebidas alcohólicas, pueden disparar a civiles o intentar forzar a una mujer a tener relaciones sexuales. A diferencia de otros juegos con temática bélica, en This War of Mine los soldados dan voz de alerta y amenazan a un personaje antes de arribar a una actitud violenta, pero si estas advertencias se ignoran esto se genera, los soldados dispararán contra el civil sin más.

En este marco, el jugador modelo de This War of Mine se encuentra inmerso en la tarea de volver funcional un grupo de individuos cuyo encuentro en el conflicto es la mayor parte de las veces fortuito, y que poseen apreciaciones diferentes en su forma de resignificar el entorno. Los esquemas simbólicos previos al conflicto y la esperanza en el restablecimiento de la normalidad operan como un puente entre los personajes, mientras que la imposibilidad de fundar nuevas formas de vincularse que se desprende del estado de excepción en el que se encuentran inmersos constituye un obstáculo para la consolidación de vínculos estables.

\section{REPRESENTACIÓN HISTÓRICA EN EL ENTORNO DE JUEGO}

El historiador Eric Hosbawn planteaba que se hacía historia desde abajo «sólo a partir del momento en que la gente corriente se convierte en un factor constante en la toma de grandes decisiones y en tales acontecimientos. No sólo en momentos de excepcional movilización popular como, por ejemplo, las revoluciones, sino en todo momento o durante la mayor parte del tiempo» (Hosbawn, 2002:206). Este concepto histórico permea el diseño del videojuego This War of Mine, que coloca al jugador en la posición de un civil atrapado en una zona de guerra y que debe hacer todo lo posible por sobrevivir. El mundo simulado de la ciudad Peygorem, con sus edificios en ruinas y habitados por recelosos supervivientes, es una referencia clara a guerras contemporáneas. La idea para el juego le surgió al ceo de 11 bit Studio, Grzegorz Miechowski, luego de leer artículos sobre lo que la gente común puede hacer para sobrevivir a la guerra (Preston, 2015).

Ciertas partes del juego están inspiradas en la experiencia directa del sobreviviente Emir Cerimovic, que vivió su infancia en Sarajevo y, además de brindar su testimonio, participa en uno de los trailers del juego. El asedio de Sarajevo duró desde el 5 de abril de 1992 hasta el 29 de febrero de 1996 y fue uno de los conflictos más largos y violentos de la historia moderna, con soldados y francotiradores disparando a hospitales y filas de civiles en mercados y destruyendo la infraestructura básica para causar hambre y sed como estrategia de guerra (Locke, 2009: 1-12).

Aunque deliberadamente se ha buscado evitar basar el juego en un conflicto específico para lograr que cualquier jugador pudiera sentirse identificado y universalizar el mensaje del juego, el diseño y la estética se inspiran principalmente en la Guerra de Bosnia y Herzegovina debido a la abundante cantidad de documentación disponible. También sobre los conflictos 
en Varsovia durante la invasión nazi y la invasión rusa a causa de formar parte de la historia familiar de los desarrolladores (Preston, 2015).

En menor medida, se tuvo en cuenta al conflicto sirio en Aleppo, a Monrovia en Liberia, Palestina, y otros sucesos bélicos sucedidos en el siglo XX e inicios del XXI. Para lograr un mayor realismo se entrevistó a supervivientes de Sarajevo y Aleppo buscando respetar, a grandes rasgos, las peripecias que sufrieron los civiles atrapados en dichos conflictos (Guillermo, 2015). Algunos de estos supervivientes intervinieron en pruebas de testeo del juego incorporando sus sugerencias en el gameplay final.

El esfuerzo de los desarrolladores por plasmar la complejidad del entorno que presenta la guerra para los civiles en el juego a nivel de diseño, en estética y especialmente en las mecánicas, ha llevado a que sea considerado un hito por la prensa especializada, obteniendo los premios "al elegido del público" y "a la excelencia narrativa" en el Independent Games Festival de 2015. El historiador y diseñador de juegos del Gilles Roy afirmó que el juego «nos invita a asomarnos al abismo del registro histórico para escuchar voces que no se han tenido en cuenta adecuadamente, excepto tal vez en la ficción histórica» (Roy, 2016).

Cabe señalar que This War of Mine no ofrece al jugador tutorial alguno ni guía de ningún tipo al empezar a jugar. Tampoco permite grabar la partida y volver a intentarlo. Las acciones y sus consecuencias en el juego se muestran siempre irreversibles. La mecánica denominada "muerte permanente" (perma-death) suma realismo al juego y refuerza la idea de que la guerra atrapa al civil desprevenido y no existen segundas oportunidades. En palabras del director Przemek Marszal, «no hay tutorial porque cuando la guerra se desata no hay un tutorial de gente diciéndote que hacer para sobrevivir y salvar a tu familia. Estas por tu cuenta» (Hudson, 2015).

Los trastornos de estrés postraumático, angustia, pesadillas y suicidio en los supervivientes debido a los sufrimientos de las guerras han sido ampliamente documentados (Summerfield, 1995 y Kraft, 2006). La mayoría de la gente que juega por primera vez pierde en poco tiempo, con sus personajes agobiados por el hambre, las enfermedades, la depresión o la violencia. La muerte de un personaje miembro del grupo causa la inmediata depresión en los otros e incluso el asesinato de un individuo externo puede llevar al suicidio de otros. Esta mecánica representa la angustia detectada en los relatos de los supervivientes de la guerra y, de acuerdo a la perspectiva de autores como Diego Mate, el juego «suma una dimensión humana al acto de representar la muerte, exhibe el dolor en los otros, la culpa, el arrepentimiento, el recuerdo» (Mate, 2015).

No obstante, a través de intentarlo repetidamente el jugador puede establecer las cosas que es necesario hacer para que los personajes vivan. Las mecánicas para lograr este objetivo, como la manufactura de objetos, obtener agua hirviendo la nieve y calor quemando muebles o el intercambio de comida y medicamentos por armas, alcohol y cigarrillos también están basadas en las formas de sobrevivir que relataron los sobrevivientes en las entrevistas para el juego y/o que han sido recogidas por especialistas en los documentos históricos.

El marco referencial de This War of Mine puede observarse tanto en sus componentes estéticos como en sus componentes normativos, la gran cantidad de para-textos que ofrece el juego tanto en las frases que dicen los personajes que maneja el jugador, otros personajes con los que éste puede interactuar, así como en los detalles gráficos de los escenarios.

Respecto a los componentes estéticos, el juego se presenta con un estilo gráfico sumamente realista, con fondos ricos en detalles y personajes de proporciones correctas, matizado con colores oscuros y gamas de grises que subvierten ligeramente las 
representaciones para enfatizar la atmosfera oscura y opresiva. El impacto de la gama de colores causa la ilusión de desesperanza y desesperación, mientras que la interrupción de la banda sonora por ocasionales estadillos y ruidos de mortero transmite la sensación de angustia (Ecenbarger, 2016).

En algunas de sus expansiones se utilizan animaciones y fotografías que imitan a las filmaciones de los corresponsales de guerra y la propaganda bélica. Es interesante remarcar que muchos de estos elementos estéticos que se muestran en los escenarios están basados en hechos reales sucedidos en diferentes teatros de guerra. Entre estos se incluyen:

1. Un edificio de torre de oficinas envuelto en llamas y humo cuyo diseño imita a la conocida foto del bombardeo sobre el edificio de la Amistad entre Gracia y BosniaHerzegovina en Sarajevo, tomada por el fotógrafo ruso Mikhail Evstafiev durante la guerra de Bosnia y Herzegovina en la primavera de 1992.

2. La cita de Hemingway Fuck this War fue incluida en el juego debido a su eficacia para representar lo horrible que es la guerra, pero la fuente en que está escrito y su posicionamiento en la pared exterior de la casa en la que se encuentran los personajes hace referencia al grafiti, que puede hallarse aún hoy en una casa derruida y abandonado en Mostar, utilizado durante el conflicto bélico como refugio por sobrevivientes.

3. Algunos ejemplos de paratextos contienen expresiones como "Antes de la guerra» y «Cuando esto termine...» que remiten a los tiempos de paz como lo normal y a la guerra como la interrupción de la normalidad. La historiadora Ivana Maček ha documentado el uso del concepto de normalidad en los sobrevivientes de Sarajevo para referirse no sólo al modo de vida anterior a la guerra sino al marco moral que guía sus acciones, estableciendo que una vida normal representa el modo en que la gente desearía vivir y que una persona normal piensa y actúa de forma aceptable (Macek, 2009:5-6).

En cuanto a los elementos normativos que componen el juego, encontramos una doble construcción mimética dentro del sistema de normas que regulan los personajes y objetos. El jugador maneja un grupo de personajes que están expuestos a los flagelos como (a) las inclemencias climáticas, (b) las enfermedades y las heridas, (c) el hambre, la depresión y (4) el terror a los ataques de los soldados e incluso otros supervivientes.

Como consecuencia de la representación de estas situaciones el sistema de reglas obliga no sólo a generar un conjunto de interacciones positivas entre los personajes (diálogos, abrazos, consuelo) para mantener la moral sino también a obtener ciertos objetos (comida, suministros, medicamentos, herramientas y armas) que oficien de protección frente a los peligros del juego y que deberán ser construidos a partir de los recursos básicos que puedan saquearse en los edificios o intercambiarse. En estas interacciones también se encuentran referencias históricas a hechos reales. El valor como objeto de intercambio del tabaco en la Segunda Guerra Mundial y de bebidas alcohólicas durante el conflicto de Kosovo aparece con frecuencia en testimonios de sobrevivientes, mercenarios y soldados que estuvieron en el frente. La inclusión de estos elementos específicos en la dinámica del juego es una decisión deliberada de los desarrolladores (Guillermo, 2016).

Algunas situaciones como violaciones, saqueos y asesinatos son habituales en los conflictos bélicos y se ha comprobado su impacto en la psique de los sobrevivientes (Summerfield, 1995). En el videojuego son representadas en forma verosímil, presentando 
al jugador la posibilidad de intervenir en diversas maneras. La inclusión de decisiones moralmente ambiguas y el amplio grado de inmersión e identificación con los personajes coloca al jugador en una posición difícil. Esta ambigüedad se utiliza como el recurso principal del gameplay para crear una experiencia "ética" de juego, forzando a los jugadores a deliberar y reflexionar sobre sus propias acciones (Toma, 2015).

A partir de estos elementos se observa en This War of Mine un potencial didáctico notable para abordar las guerras contemporáneas mediante la experiencia de juego en la que el jugador puede identificarse con las situaciones vividas durante la guerra por la población civil cercana al frente y enfrentarse a las decisiones morales relacionando causas y consecuencias en situaciones que son representativas de aquellas acaecidas durante la guerra.

Otra posibilidad es ilustrar los tipos ideales construyendo un actor colectivo histórico mediante la agregación de las conductas de los personajes de las historias individuales. Por su parte, el historiador G. Roy sugiere que «una recreación ficticia basada en la evidencia de la supervivencia en tiempo de guerra como This War of Mine, nos permite examinar los mecanismos básicos, o los 'requisitos mínimos', si se quiere, de supervivencia y cohesión grupales» (Roy, 2016).

Por último, es interesante notar que el editor incluido en el juego habilita la posibilidad de generar contenido, lo que permite explorar diferentes posibilidades y refuerza en cierto sentido el potencial del juego como simulador social.

\section{ALGUNAS OBSERVACIONES SOBRE EL DISCURSO IDEOLÓGICO DE THIS WAR OF MINE}

Como afirma Miguel Sicart, el discurso moral o ideológico que presenta un juego respecto de los elementos que incluye se vincula a la forma en la que establece una valoración de los mismos a partir de su sistema de bonificaciones y penalizaciones. En este sentido juegos como This War of Mine conllevan un discurso ideológico que tiende más bien a problematizar la valoración de los elementos, ofreciendo a los jugadores un escenario en el que deben optar por diferentes posibilidades sin poder anticipar de un modo mecánico y claro el resultado de las mismas. En este sentido, el valor de las acciones dentro del juego es difícil de anticipar para los jugadores, pues acciones como robar o cometer un crimen pueden otorgar beneficios materiales. Al mismo tiempo, exponen a los personajes a riesgos y pueden alterar su estado anímico de un modo negativo, llevándolos a un estado en el que ya no sean capaces de realizar tareas. La resistencia a cometer estos actos para asegurar la subsistencia también puede ocasionar que el jugador no logre proveer a los personajes de los insumos mínimos necesarios y pierda la partida.

Esta ambigüedad del valor de las acciones, en las que el personaje/jugador debe encontrar cuál es su límite moral frente a determinadas acciones, encuentra excepciones que permiten entrever algunas líneas del discurso ideológico del juego que operan en la relación que establece el sistema de penalizaciones y bonificaciones frente a algunos elementos de la mecánica de juego. Sobre ello cabe mencionar tres aspectos que ofrecen dentro del juego algunas huellas de un imaginario capitalista dentro de la mecánica de juego. Estas se muestran hasta cierto punto discordantes con las experiencias que explicitan los creadores del juego. 
1. El primer aspecto es el hecho de que el juego no penaliza de ningún modo la realización de cambios ventajosos. Si otro sobreviviente, por ejemplo, necesita medicamentos porque tiene a un familiar enfermo y el jugador se los ofrece a cambio de la mayor cantidad de objetos que éste pueda ofrecer, esto no conlleva ninguna consecuencia negativa ni es penalizado de ningún modo por el esquema moral del juego. Ni en relación a la mecánica ni en relación a los elementos para-textuales, con excepción de algún matiz en la respuesta del personaje con el que se realiza el intercambio. Incluso cuando el juego propone en muchas ocasiones la posibilidad de ayudar a otros sobrevivientes o de regalarles medicamentos $u$ otros elementos para asegurar su supervivencia, este altruismo no presenta un obstáculo al afán de lucro en los intercambios. El personaje/jugador puede tomar ventaja de las necesidades extremas de otro personaje a su favor, sin que ello afecte a su estado anímico en absoluto o tenga alguna consecuencia negativa. Al mismo tiempo, la generosidad que es valorada por la mecánica de juego cuando se ayuda a otros personajes carece de efecto positivo en los intercambios.

2. En segundo lugar, incluso cuando el estado anímico o de cansancio pueden volver a los personajes disfuncionales para realizar cierto tipo de tareas, ya sea por la lentitud que muestran los personajes cansados o por el peligro de arribar a un estado de depresión, los personajes pueden incrementar su estado de hambre o de enfermedad, incluso hasta morir sin que ello posibilite que tomen por sí mismos algún objeto del inventario del juego. Los personajes pueden sentarse, fumar, desmayarse o realizar algunas tareas de manera automática, pero ningún estado de ánimo, ni de hambre extrema o de enfermedad evitan que el jugador posea un total control de la administración de los recursos disponibles. Este aspecto, asociado a la maximización del uso de los recursos, promueve una administración de los recursos que incorpora el hambre, el cansancio, el frío, el estado anímico y el grado de enfermedad de los individuos que componen el grupo como elementos que pueden administrarse para optimizar el rendimiento del grupo y sus resultados. Esta posibilidad se hace eco, sin duda, de la lógica de maximización y optimización de los recursos disponibles, que tiende a cosificar o mercantilizar incluso el bienestar de los operarios en el sistema neoliberal. En este sentido pueden recuperarse las referencias de autores como Óliver Pérez-Latorre en relación a una representación de una precarización cool o una presentación algo romanizada de la precarización en los videojuegos contemporáneos (Pérez Latorre et al. 2017).

3. En tercer lugar, y también asociado a determinados aspectos del imaginario capitalista que envuelven al juego, se observa una tendencia en el mismo a dar un lugar central y tal vez algo exagerado a la producción de tipo fabril al interior del refugio de los supervivientes, así como a la posibilidad de especular, a partir de las noticias que la radio ofrece sobre la escasez de insumos como el café o los cigarrillos en la ciudad para mejorar las condiciones de comercio. Esta perspectiva del refugio como una pequeña fábrica o empresa se ve reforzada por la posibilidad de utilizar cualquier personaje para cualquier tarea. La falta de especificidad de conocimiento para realizar las tareas ofrece en los procesos de fabricación o construcción sólo una variable cuantitativa en relación a la necesidad de un operario y una cantidad de tiempo. El tiempo de realización puede ser mayor o menor dependiendo del estado del personaje, pero no tiene relación con ningún aspecto particular del mismo. En este 
sentido, se prioriza una perspectiva pragmática de administración de las acciones en la que los personajes se muestran como recursos equivalentes para cualquier tarea de producción.

Estos tres aspectos que pueden observarse en cierta forma como la expresión de contenidos ideológicos que se muestran algo discordantes con el conjunto de contenidos del juego, y con las intenciones que sus autores han explicitado respecto del mismo, coinciden con aquello que autores como Clint Hocking han denominado disonancia lúdico-narrativa (2007), que se identifica con la fricción que puede producirse entre el contenido narrativo de un juego y aquellos contenidos simbólicos que su mecánica sugieren, lo que ha sido mencionado también por otros autores como un conflicto entre el mensaje del videojuego y sus aspectos procesuales (González Tardón, 2014: 118-123). Esta fricción entre la mecánica de juego y su esquema simbólico es recuperada por Pérez Latorre como un elemento clave para la apropiación del discurso ideológico inscripto en el videojuego, pues lejos de reducirse a un fallo de diseño que genera una desnaturalización respecto del esquema narrativo del juego, o que irrumpe el carácter inmersivo del entorno lúdico, muestra el carácter ambiguo y problemático del discurso ideológico que presentan los videojuegos como productos culturales que proyectan una serie de contenidos simbólicos particulares, pero que se encuentran inmersos en un marco simbólico más amplio y que reflejan huellas o ecos de ese imaginario cultural al que pertenecen (Pérez Latorre et al., 2017, Pérez Latorre, 2018).

En este sentido, cabe recuperar el entorno de juego de This War of Mine como un entorno complejo que no puede ser comprendido desde una perspectiva unilateral, en la medida que propone a los jugadores desarrollar acciones cuya relación con las condiciones de victoria se muestra confusa a lo largo del juego, a la vez que obliga a resignificar elementos conocidos por el personaje/jugador en un nuevo esquema de valores caracterizado por la excepcionalidad frente a las formas sociales de hacer significar del mundo contemporáneo. Esta ambigüedad se muestra reforzada a su vez por la presencia en la mecánica de juego de elementos propios del imaginario social vinculado al discurso neoliberal que pueden destacarse tal como se ha realizado en este último apartado, y que no parecen afectar en absoluto el estado anímico de los personajes, mostrándose en cierta forma como puntos ciegos del entorno moral que el playworld busca representar, a la vez que suscriben el esquema simbólico del juego al imaginario social contemporáneo.

\section{BIBLIOGRAFÍA CITADA}

AARSETH E.J., (2007) "Aproximaciones metodológicas al análisis de videojuegos", Revista Artnodes. $\mathrm{N}^{\circ} 7$.

BOGOST I., (2007) Persuasive games. The Expressive Power of Videogames. Cambridge. MIT Press.

CASTORIADIS C., (2004) Sujeto y verdad, Bs. As., Fondo de Cultura Económica.

- (2005a) "Lo imaginario: la creación en el dominio histórico social" en: Los dominios del hombre, Barcelona, Gedisa.

- (2010) La institución imaginaria de la sociedad, Bs. As., TusQuets.

ECENBARGER, C. (2016). "In war, not everyone's a soldier." A Review of This War of Mine. Press Start, 3(2), 70-73. Recuperado el 21/03/18 de http://www.press-start.gla.ac.uk/ index.php/press-start/article/view/48/49

Quaderns, 13 (2018), pp. 23-36 
HOCKING C., (2007) "Ludonarrative dissonance in Bioshock: The problem of what game is about" en: http://clicknothing.typepad.com/click_nothing/2007/10/ludonarrative-d.html

HOBSBAWM, E., (2002) Sobre la Historia. Barcelona, Editorial Crítica.

HUDSON L., (2015) "Life as a Target" recuperado el 20/03/18 de: http://www.slate.com/ articles/arts/gaming/2015/02/siege_survival_video_game_this_war_ofmine_reviewed.html HUIZINGA J., (1972) Homo ludens. Madrid, Alianza. 2012.

FRAILE J. A., (2012) Bosquejo de una metafísica del videojuego, España, Círculo Rojo.

FRASCA G., (2009) "Juego, videojuego y creación de sentido", en: Revista Comunicación No 7. Pp. 37-44

GONZÁLEZ TARDÓN C., (2014) Videojuegos para la transformación social. Aportaciones conceptuales y metodológicas. Tesis Doctoral. Universidad de Deusoto.

GUILLERMO G. M., (2016) "Entrevista a Pawel Miechowski, senior writer de 11 Bit Studios", sitio web Deux Ex Machina Videogames Magazine, recuperado el 20/03/18 de http:// deusexmachina.es/entrevista-a-pawel/

JUUL J., (2011) Half-Real: Video Games between Real Rules and Fictional Worlds, Cambridge, The MIT Press.

KRAFT R. (2006) Archival Memory: Representations of the Holocaust in Oral Testimony, Poetics Today. 27 (2): 311-330.

LOCKE P., (2009) City of survivors Trauma, grief, and getting by in post -war Sarajevo, Tesis para Doctorado en Filosofia, Princeton University.

MAČEK I (2009) Sarajevo Under Siege: Anthropology in Wartime. Filadelfia: Universidad de Penn.

MANOVICH L., (2001) The Language of new media. Cambridge, MIT Press.

MARCANO B., (2008) "Juegos serios y entrenamiento en la sociedad digital." en: Teoría de la Educación. NN 3. Salamanca. Pp. 93-107

MARTí J.P., (2011) "Aproximación metodológica al análisis de videojuegos publicitarios", en: Revista deSignis, No17, Bs As, Pp. 133-141

MATE D. (2018), "La representación de la muerte en el videojuego", en: Jangwa Pana: Revista de Ciencias Sociales y Humanidades, Vol. 17, No.1

MCGONIGAL J., (2011) ¿Por qué los videojuegos pueden mejorar tu vida y cambiar el mundo?, Bs. As., Siglo XXI. 2013.

NAVARRETE C., (2014) "El pensamiento abductivo como fundamento ontológico de los videojuegos", en: Icono 14, vol.12. pp. 416-440.

PARKIN S., (2015) Muerte por video-juego, Madrid, Turner, 2016.

PÉREZ LATORRE Ó., (2010) Análisis de la significación del videojuego. Fundamentos teóricos del juego, el mundo narrativo y la enunciación interactiva como perspectiva del estudio del discurso. Tesis Doctoral. UPF.

- (2018) "El videojuego en el escenario de la cultura mainstream. Repensar al videojuego como producto cultural y su inserción en el imaginario contemporáneo." (Entrevista realizada por Emiliano Aldegani) en: Revista E-Tramas $N^{\circ} 1$, En prensa.

PÉREZ LATORRE Ó; OLIVA M., (2017) "Video games, Dystopia and Neoliberalism: The Case of BioShock Infinite" en: Games and Culture. Pp. 1-21

PÉREZ LATORRE Ó; NAVARRO V; PLANELLS A; SÁNCHEZ C., (2017) "Recessionary games: Video games and social imaginary of the Great Recession (2009-2015)" en: Convergence: The International Journal of Research into New Media Technologies. Pp. 1-17. 
PLANELLS A. J., (2015) Videojuegos y mundos de ficción. De "Super Mario" a "Portal". Cátedra, Madrid.

PRESTON D. (2015) "Interview: Pawel Miechowsky of this war of mine \& 11 bit studios", Outerweb blog, recuperado el 20/03/18 de http://outermode.com/interview-pawelmiechowski-this-war-of-mine-11-bit-studios

RAMÍREZ J. A., (2013) Análisis narratológico del videojuego documental. Tesis de Maestría. UNAM. ROY G., "This War of Mine: Human Survival and the Ethics of Care" en: Play The Past, blog recuperado el 20/03/18 de http://www.playthepast.org/?p=5618

RUIZ D., (2010) Aprendiendo con videojuegos: jugar es pensar dos veces. Madrid, Ministerio de Educación. Narcea Ediciones.

RUIZ S. D., (2009) "El videojuego como herramienta de comunicación publicitaria: una aproximación al concepto de advergaming", en: Revista Comunicación, Nº 7.

SALEN K., y ZIMMERMAN E., (2006) Rules of Play: Game Desing Fundamentals. Cambridge. MIT Press.

SÁNCHEZ MURCIA P., (2002) "Aprendiendo habilidades con videojuegos" en: Revista Comunicar, No 19. Pp. 114-119.

SCOLARI C. A., (2008) Hipermediaciones. Barcelona, Gedisa.

- (ed.) (2013) Homo videoludens 2.0. De Pac-man a la gamificación, Universitat de Barcelona. SICART M., (2009) The ethic of Computers games, Cambridge, MIT.

SUMMERFIELD D. (1995) "Assisting Survivors of War and Atrocity: Notes on 'Psychosocial' Issues for NGO Workers" en: Development in Practice Vol. 5, No. 4. Pp. 352-356.

TOMA E., (2015) Journal of Comparative research in anthropology and sociology, Volume $6, N^{0} 1$.

WOLF P., (2005) "Introducción a la teoría del videojuego", en: Formats. N 4. Barcelona. 